\title{
Implementación de un sistema presencial y virtual para la incubación y aceleración empresarial
}

\section{Implementation of a in place and virtual business incubation and acceleration system}

Arguedas Alfaro, Rebeca

Universidad Internacional del Ecuador, Ecuador

Autor para correspondencia: rebeargo82@gmail.com

Fecha de recepción: 01 de Junio de 2017 - Fecha de aceptación: 15 de Agosto de 2017

Resumen: La implementación de un sistema presencial y virtual para la Incubación y Aceleración Empresarial ofrece a los nuevos emprendedores un apoyo y acompañamiento para formar y /o acelerar empresas con un alto valor agregado mediante la innovación y capacitación, propiciando que estén presentes en el mercado por largo tiempo. Se realizaron diferentes estudios del entorno para conocer y sustentar la vialidad de la implementación de este servicio a los emprendedores. Se determinan las empresas que actualmente se encuentran en esta línea de negocio, se revisan sus metodologías de trabajo para dar una base sólida a la propuesta de servicios. Existe una gran oportunidad de apoyar a los emprendedores ya que se estima que entre un 70-80\% de los emprendimientos fracasan tras el primer año de vida y quienes han subsistido terminan muriendo pasados 4 o 5 años, siendo una cantidad marginal las que sobreviven. Hoy, para que una idea emprendedora proporcione frutos es necesario un acompañamiento, un sistema de apoyo que ayude y fortalezca esa idea.

Palabras clave: aceleradora; emprendedor; incubadora; sistema bimodal; pyme

\begin{abstract}
The creation of a in place and virtual business incubation and acceleration system offers novel entrepreneurs support and accompaniment to build enterprises with high added value through innovation and training, encouraging their long-lasting presence in the market. Different studies were conducted of the environment to determine and sustain the feasibility of the implementation of this service for entrepreneurs. Businesses that are currently in this line of operation are defined, and their operating methods are reviewed to provide solid foundations to their service proposal. Great opportunities exist to support entrepreneurs since it is estimated that between 70 and $80 \%$ of enterprises fail after the first year of operations, and those that survive last 4 or 5 years, with a very slim margin that remain. Today, accompaniment is necessary for a business idea to thrive, a support system to help and strengthen that idea. The project to create a bimodal business incubation and acceleration system is feasible, with an acceptable revenue flow and financial indicator such as the NPV and IIR reflecting positive results, the initial business investment is relatively low and several external services may be hired, such as renting facilities for the offices, classrooms and equipment. A high demand can be foreseen since business projects are being born every day, with known specific needs, according to the information provided by an expert panel convened for that purpose.
\end{abstract}

Key words: accelerator; bimodal system; entrepreneur; incubator; sme 


\section{Introducción}

\section{Problema o argumento}

El objetivo de esta investigación es la implementación de un sistema bimodal (virtual y presencial) de incubación y aceleración empresarial con el fin de apoyar a los emprendedores con acompañamiento y capacitación.

El país ha abordado el tema del emprendimiento y la necesidad de impulsarlo, pero en la etapa de acompañamiento y potenciación, el crecimiento de los emprendimientos todavía está en ciernes y es por esto que nace la necesidad de generar información y medir la posible implementación de un sistema para este efecto.

Las incubadoras trabajan con emprendimientos en sus etapas iniciales, cuando el proyecto es apenas una idea. Una incubadora es una compañía que se encarga de buscar productos o servicios emprendedores que posean potencial para salir al mercado. Para ello cuenta con expertos y asesores que trabajan, junto con los directivos de cada una de estos, desarrollando sus proyectos hasta que toman forma.

A diferencia de la incubadora, la aceleradora se dedica a accionar un proyecto que ya se encuentra en desarrollo. Su principal objetivo es el de obtener beneficios reales con la empresa ya establecida en el mercado. Un proceso de aceleración se vale de técnicas ágiles para facilitar la transición de ideas sólidas a hechos reales. El trabajo del acompañamiento es esencial para que el proyecto avance con una mayor celeridad.

Estas iniciativas apoyan a las empresas emergentes con capacitaciones, asesoría, acompañamiento, redes de contactos, provisión de espacio físico, entre otros beneficios.

Como ejemplos importantes, por su nacimiento desde un emprendimiento y que han sobrepasado fronteras se pueden señalar:

Tecnológico de Monterrey: Desde su fundación, ha sido una institución reconocida por su excelente formación universitaria y por haber sembrado la cultura emprendedora en México a través de las incubadoras sociales promoviendo microempresas dentro de la economía formal.

Entre otras: NXTP Laps: Ubicada en Argentina, Start-Up Chile: Es una incubadora de Chile, The Pool: También mexicana, INNpulsa: Esta incubadora es colombiana, Ingenio: Esta es una incubadora uruguaya, IncubaUC: También del país chileno, Instituto Génesis: Brasileña.

En Costa Rica: En los últimos años el país ha entrado en una etapa que tiende el apoyo e incentivo al emprendimiento con programas de crédito especializados tanto en la banca estatal como privada, pero, aun así, los últimos estudios formales que se han realizado siguen estimando que el 90\% de las MIPYMES en Costa Rica fracasan en sus dos primeros años de vida. De las más importantes se encuentra CIETEC: Los primeros pasos de la incubación y aceleración en Costa Rica se dieron en el año 1994 de la mano del Centro de Incubación de Empresas CIE, por medio de la Escuela de Administración de Empresas del Instituto Tecnológico de Costa Rica (TEC). Al principio fue una propuesta arriesgada al tratarse de un proceso desconocido para la 
institución y para el país. Luego de esta se fundan el Parque Tec , UNA Incuba, Auge, Carao Ventures, Open Future, Parque la Libertad, GN Plus.

De acuerdo al artículo "Hacia nuevas formas metodológicas en e-learning, por Salinas Ibáñez, Universidad de las Islas Baleares", Las nuevas modalidades educativas vienen ahora determinadas por la cantidad y la calidad de diálogo y las interconexiones entre profesores y estudiantes, así como por la flexibilidad del diseño de los cursos en cuanto a objetivos, estrategias de aprendizaje y métodos de evaluación. Esto supone la construcción de nuevos espacios de comunicación que posibilitan una gran variedad de situaciones comunicativas: entornos institucionales (campus virtuales, entornos virtuales), informales (redes sociales), personales, etc. Entornos que profesores y estudiantes deben acomodar, apropiarse, dominar para que se produzca el aprendizaje, la construcción personal del conocimiento, la realidad del conocimiento compartido desde los valores, creencias y experiencias personales y, desde estas premisas, el aprendizaje sea contextualizado, situado.

\section{Revisión de la literatura}

"Tenemos mucho trabajo que hacer antes de transformar una idea en negocio y de comenzar a tomar las primeras decisiones que vayan dando forma empresarial a la idea para que sea una realidad". (González, 2015). Hoy, para que una idea emprendedora proporcione frutos, es importante un acompañamiento, un sistema de apoyo, ayuda y fortalecimiento a esa idea, la información es poder, es una herramienta clave para definir estrategias.

Desde mediados de la década de los setenta y tomando en cuenta la necesidad de dar soporte y acompañamiento en la gestión de las nuevas empresas durante sus primeros años, tanto de gestación como de operación, surgen las incubadoras de empresas como instituciones dedicadas a facilitar el desarrollo de los nuevos equipos productores mediante la búsqueda de recursos financieros, la generación de contactos comerciales y la asesoría integral en la estructuración y puesta en marcha de un buen plan de negocio (Cruz y Matiz, 2004).

Quien aborda la aventura de un negocio, lo organiza, busca capital para financiero y asume todo o la mayor acción de riesgo; es este quien está realizando emprendimiento. (Silva, 2013).

De acuerdo con el informe de Estado de situación de las Pyme en Costa Rica., las PYMES que son objetivo de las acciones de política pública del MEIC representan el 75,5\% del parque empresarial que corresponde a la población objetivo para la política PYME, se compone de la siguiente manera: Las microempresas representan la mayor cantidad de empresas del parque empresarial PYME, con una participación de 70,1\%; seguidas por medianas con 16, $1 \%$ y el restante $13,8 \%$ corresponde a pequeñas.

La mayor parte de las PYMES según sector, se concentran en los sectores servicios $(43 \%)$ y comercio (41\%), seguido por industria (11\%) y $\mathrm{Tl}(5 \%)$. 


\section{Ilustración 1: Distribución Microempresas}

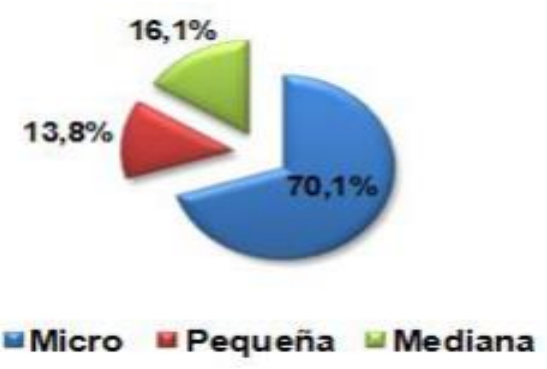

Fuente: Dirección de Investigaciones Económicas y de Mercado, MEIC con dắos del DEE-INEC.

\section{Ilustración 2: Distribución Microempresas}

A nivel educativo independientemente de si la enseñanza es presencial o a distancia, los planteamientos relacionados con la educación flexible atribuyen al alumno la posibilidad de participar activamente en la toma de decisiones sobre el aprendizaje (Salinas, 1997; Tait, 1999) y supone una nueva concepción tanto en la organización administrativa como de los materiales y sistemas de comunicación y mediación y, sobre todo, de las metodologías.

De acuerdo con el artículo del señor Pere Marqués, en la revista Educación 3.0, la revista para el aula del siglo XXI, "El currículum bimodal consiste en que al organizar las clases, los profesores tengan bien claros los conceptos imprescindibles que deben aprender los alumnos para trabajarlas de forma sistemática: algunas de ellas con ayuda de la tecnología, de forma colaborativa o con lápiz y papel”.

Miriam García Guzmán (2016) en su tesis, “Análisis sobre las potencialidades del uso de la metodología bimodal en la enseñanza de las ciencias sociales", de la Universidad UNIR (Universidad Internacional de la Rioja) dice: La sociedad actual ha cambiado y nos encontramos inmersos en una era digital que ha transformado las bases de nuestra sociedad y sigue haciéndolo apresuradamente. La educación tiene un papel muy importante a la hora de formar personas capacitadas para desenvolverse en esta sociedad, por esta razón es necesario mejorarla y adaptarla a las nuevas necesidades, creando de este modo un nuevo paradigma educativo.

La incubadora de emprendimientos, se considera como una ventana de oportunidades para la consolidación de ideas que responden a un conjunto de necesidades, crear condiciones que potencien el espíritu emprendedor, la innovación y la generación de emprendimientos empresariales y sociales que buscan nuevas formas de responder a la sociedad de manera responsable social y ambientalmente por el bienestar común de todas y todos. 


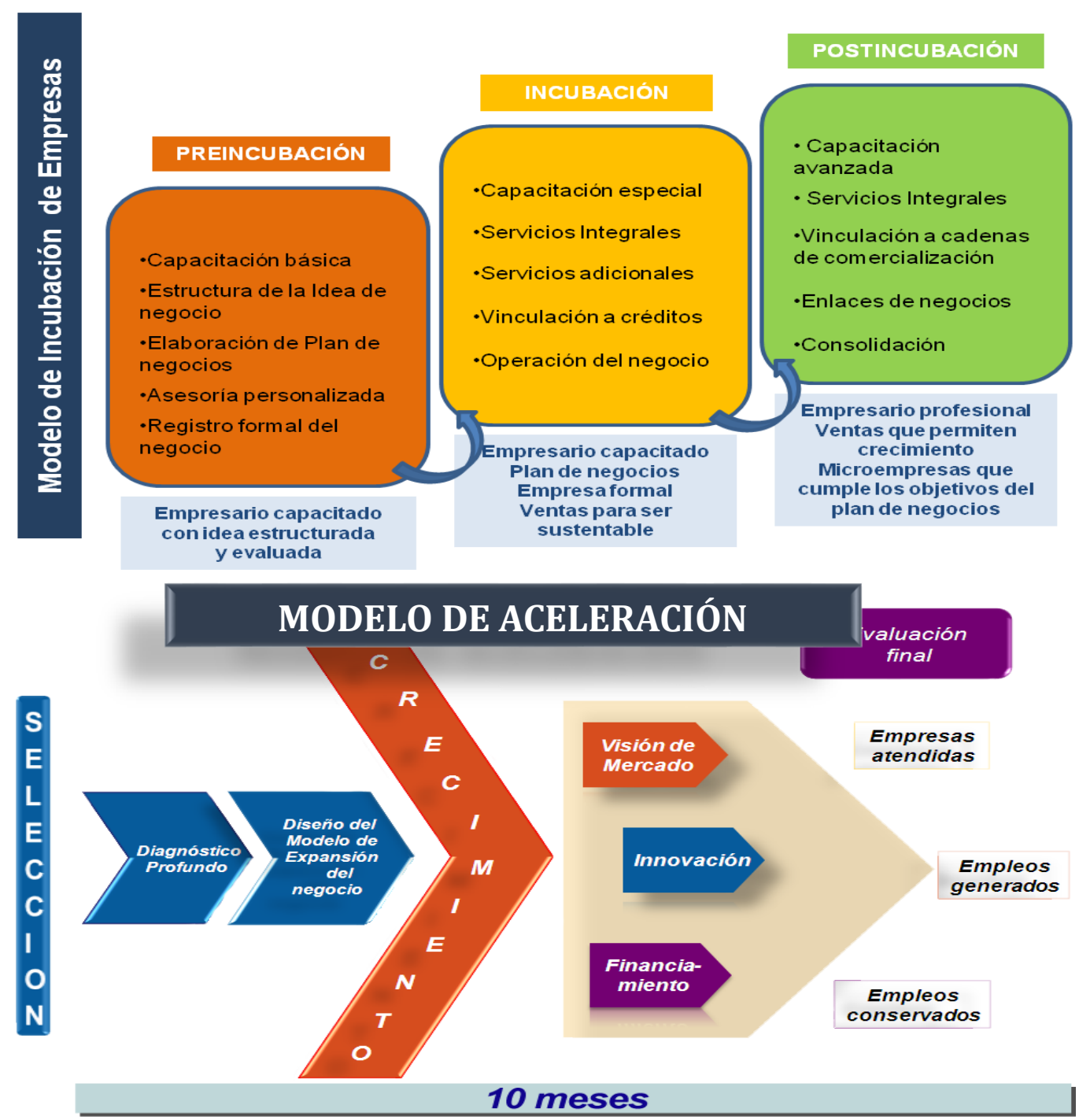

Ilustración 2. Modelo de Incubación de Empresas

Fuente: Instituto Tecnológico de Monterrey

\section{Finalidad}

Se realiza esta propuesta con el propósito estratégico de impactar al desarrollo, fortalecimiento y consolidación de los sectores empresariales y productivos mediante metodologías propias de incubación y aceleración empresarial. Adicionalmente, no hay una fuerte vinculación de grupos de ángeles inversores y mentores del sector privado. Si bien la tarea de gobierno está en la promoción del emprendimiento, hay una fuerte responsabilidad del sector privado de involucrarse en esto.

Para las nuevas empresas que son intensas en conocimiento y tecnología, se hace necesario ofrecerles procesos de acompañamiento personalizados que garanticen un ambiente propicio para la creación y fortalecimiento de las nuevas unidades productivas, es por esto que está promoviendo la puesta en marcha de incubadoras y aceleradoras de empresas en el país mediante el uso de metodologías bimodales. 


\section{Metodología}

Dada las características del estudio, los tipos de investigación utilizados son:

Enfoque cualitativo: la investigación cualitativa es aquella que utiliza preferente o exclusivamente información de tipo cualitativo y cuyo análisis se dirige a lograr descripciones detalladas de los fenómenos estudiados.

Investigación descriptiva: se utiliza el método para organizar, resumir y presentar datos de manera informativa. También de comparar resultados e interpretarlos para un mejor conocimiento de la situación. Al intentar reconocer si la inversión es rentable, definir sus pautas y comparar los efectos que se podrían obtener como resultado de este estudio, se define que este análisis es de carácter descriptivo, ya que, definirá las razones y variables que precisan la situación actual y comparará el nivel de impacto y su relación futura.

Sujetos de investigación y fuentes de información: Para obtener los datos y la información necesaria se consultó de forma directa a los expertos en temas de incubación y aceleración.

\section{Fuentes de Información}

Fuentes Primarias: Panel de expertos. Este panel se realizó con 10 expertos, cada uno de los expositores presentó su punto de vista. Así mismo, para tener la información de manera más detallada y respaldar con fundamento, el análisis de los datos se le aplicó un cuestionario.

Fuentes Secundarias: Como información complementaria se considera: libros sobre temas de emprendedurismo, finanzas y temas afines a la investigación, así como publicaciones, Red Nacional de incubación y aceleración (RNIA), Ministerio de Economía, Industria y Comercio (MEIC), Política Nacional de emprendimiento, Páginas Webs de las actuales incubadoras y aceleradoras del país.

\section{Resultados}

Se mide la viabilidad del proyecto por medio de:

- La requisición de la información que ayude a enfrentar las condiciones del mercado, tomar decisiones y anticipar su evolución.

- Se adquiere la máxima información posible sobre incubadoras y aceleradoras del país.

- Se determina la oferta, la demanda y los canales de comercialización que permitan elaborar la estrategia de marketing.

- Se fijan los precios más adecuados al tipo de servicio y exigencias de los usuarios.

- Se determina el perfil del cliente

Del panel de expertos en el tema, pertenecientes a instituciones como el Instituto Tecnológico de Costa Rica, Instituto Nacional de Aprendizaje, AUGE, Fundación Parque la 
Libertad, Avantica, AAA Servicios, Centro Ejecutivo Miraflores y la Agencia para el desarrollo de la Región Huetar Norte, se obtienen las siguientes apreciaciones:

Del total de los 10 participantes, la mitad conoce alguna aceleradora de empresas en el país y la otra mitad no sabe si existen.

Pero en su totalidad el panel de expertos si conoce las incubadoras ubicadas en el país. Dentro de las organizaciones dedicadas a este ejercicio, mencionan las siguientes:

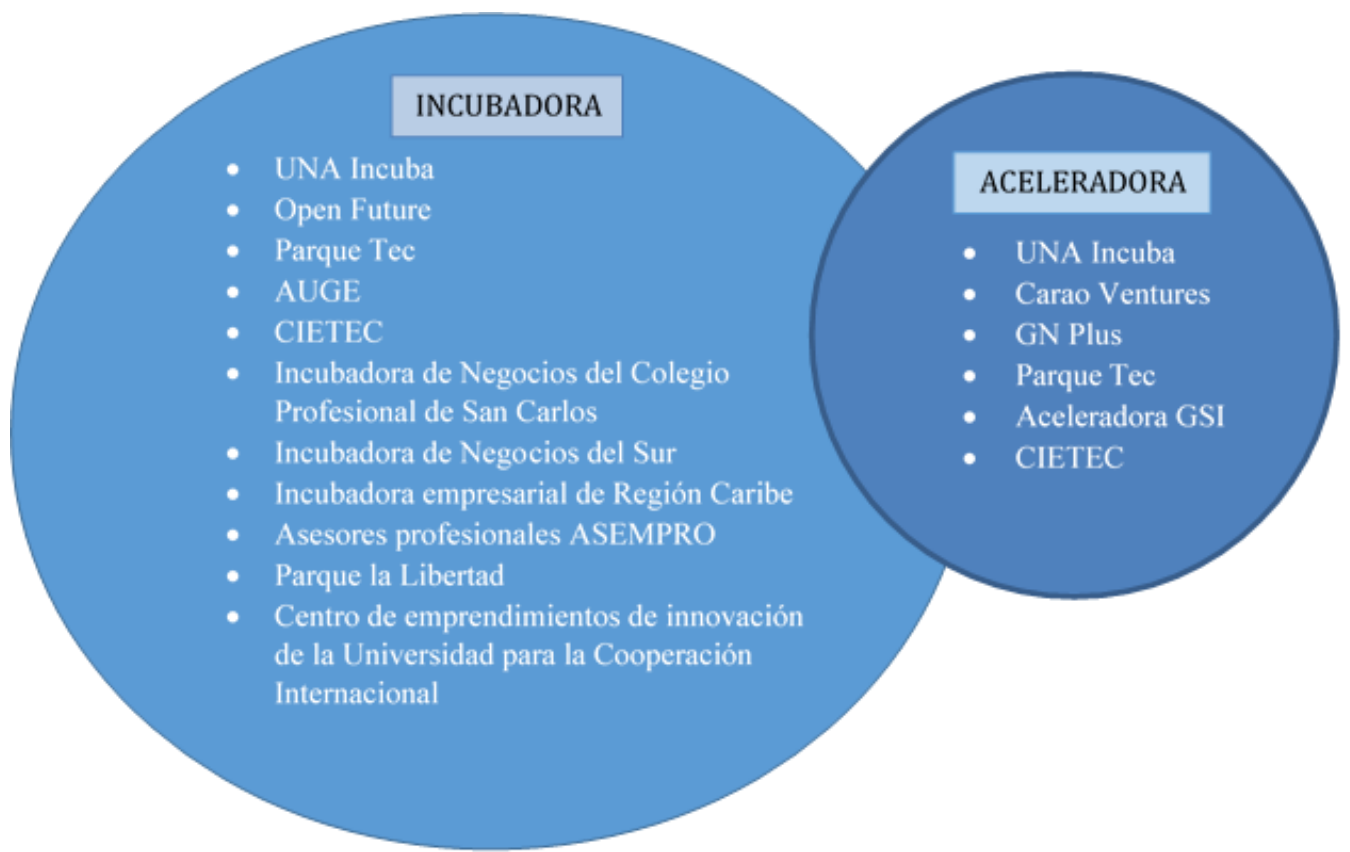

Fuente: Elaboración propia

Las incubadoras más mencionadas por los asistentes en el panel son las de CIETEC y Parque TEC. Enfatizando así que actualmente el CIETEC, de acuerdo con el panel de expertos es el más reconocido tanto como incubadora como aceleradora a nivel país.

Durante el panel realizado con los expertos, se realizó la pregunta de cuáles deberían de ser los objetivos primordiales de estas empresas como incubadoras y aceleradoras de emprendimientos. La respuesta se presenta en el grafico siguiente: 


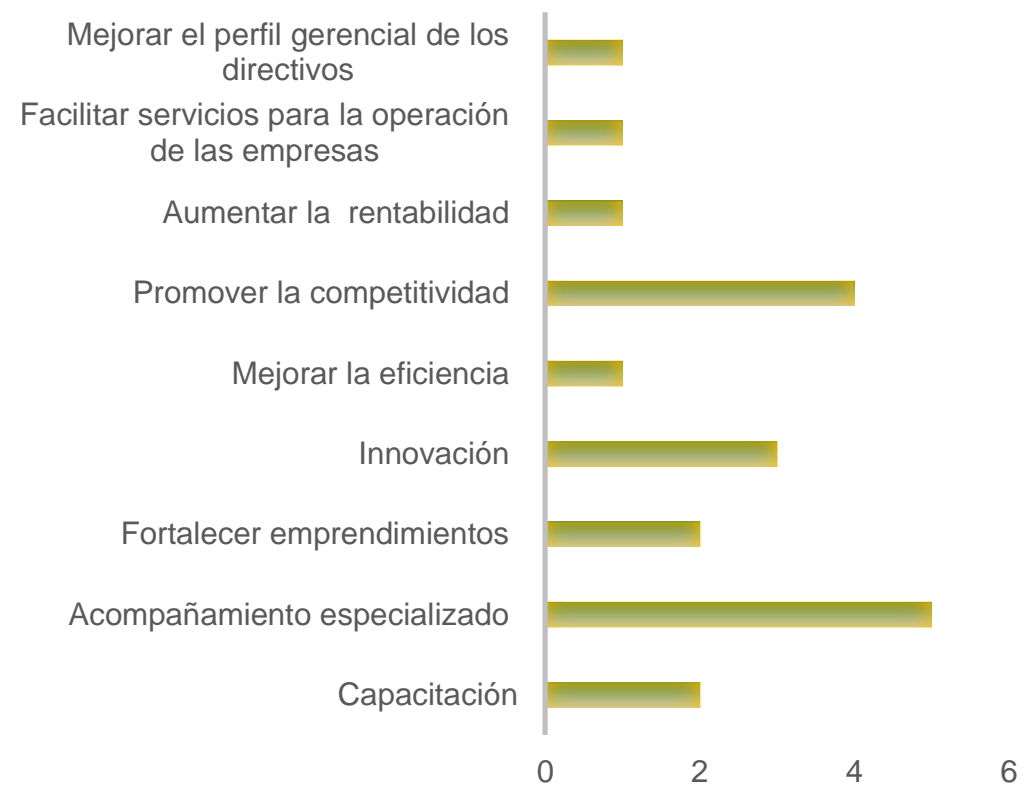

Gráfico 1: ¿Cuáles considera usted deberían de ser los objetivos primordiales de estas empresas?

Fuente: Elaboración propia

De acuerdo con lo expresado por los expertos, existen de manera generalizada 9 áreas de en las que deben fundamentarse los objetivos de una incubadora y/o aceleradora, y resaltan sobre ellas los servicios de promover la competitividad y el acompañamiento especializado.

Adicionalmente se consultó cuáles son los servicios que podrían tener mayor acogimiento dentro del mercado, de lo que se puntualiza:

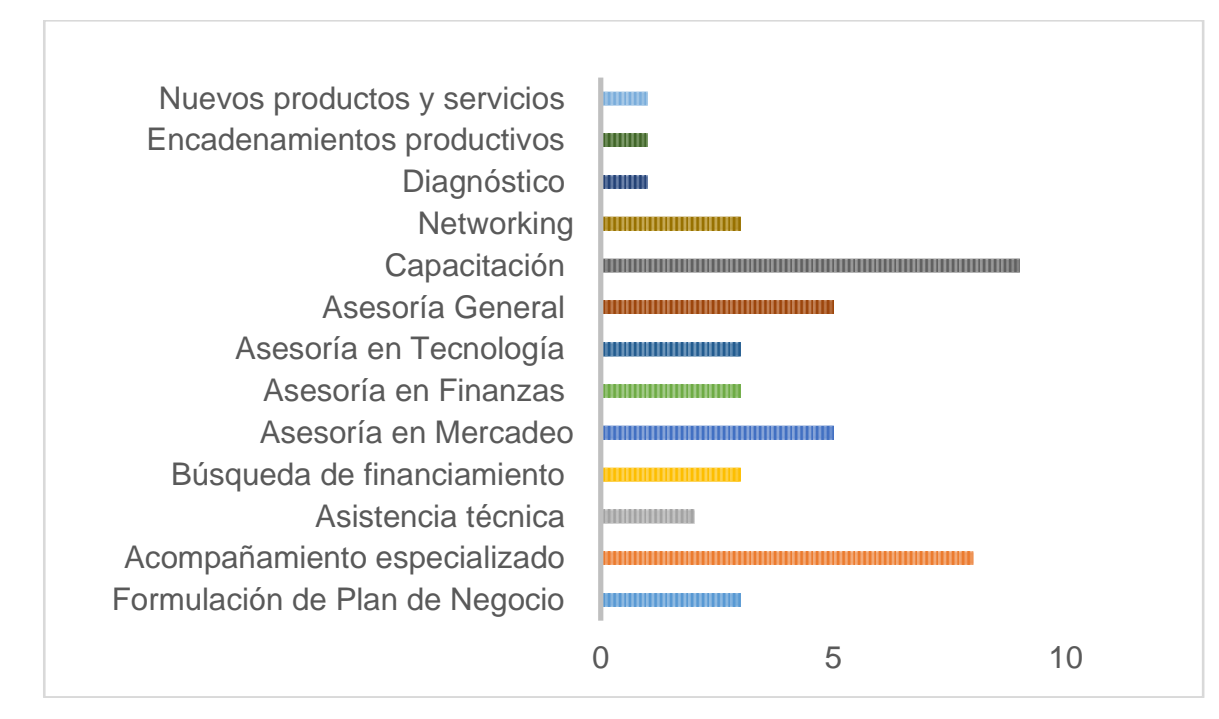

\section{Gráfico 2: ¿Cuáles serían los servicios que usted buscaría obtener de una aceleradora y/o incubadora bimodal?}

Fuente: Elaboración propia 
En este gráfico se nota, que los servicios que mayor demanda podrían tener son los relacionados con la capacitación, el acompañamiento especializado y asesoría.

Es por esto que, para dar inicio a la creación del negocio, se visualiza el impartir primeramente los módulos de:

- Modelo de negocio y plan estratégico.

- Capacitación especializada al personal.

- Herramientas informáticas aplicadas al negocio.

Así como la Asesoría en diferentes áreas administrativas como lo son:

- Diseño de la imagen corporativa de una empresa.

- Asesoría en sistemas financieros y contables aplicables al negocio.

- Asesoría legal y comercial.

- Elaboración de estudios de mercado.

- Gestión de procesos.

- Asesorías en Talento Humano.

Dentro de las consultas hechas, se realizó la de cuáles son los elementos que más influyen para adquirir un servicio como este y destacan los siguientes:

- Servicio al cliente

- Confianza

- Responsabilidad

- Eficiencia

- Compromiso

Otros que también intervienen y son importantes mencionar son:

- Calidad

- Experiencia

- Buenas prácticas

Sobresale que los diez criterios obtenidos a la pregunta de si este podría ser un servicio que se brinde de manera virtual, los diez respondieron de manera afirmativa; no obstante, indican que no en su totalidad, que para ellos además debe existir una parte de soporte presencial ya que el proceso de aprendizaje de las personas difiere una de otra.

Se identifican los factores determinantes para obtener el lugar óptimo para la implementar el negocio, establecer la cantidad y calidad del equipo que se requiere para el negocio y el personal que se va a requerir para el funcionamiento. 


\section{Discusión}

\section{Alcance:}

Con la realización del panel de profesionales instruidos en el área emprendedora, se logran datos de preeminencia para la investigación, tales como los objetivos primordiales de estas empresas lo cual se ha podido validar en la puesta en marcha de esta iniciativa.

Conocer los procesos de incubación y aceleración realizados por algunas empresas en este mercado, adicionalmente después de la visita a Loja Ecuador se pudo conversar con conocedores de esta materia y ampliar la información de con el conocimiento de las empresas en este ámbito que haya operan.

Después de la participación en el CIICAE- UIDE 2017, se puede validar la importancia de la realización de diferentes estudios económicos, financieros, de mercado entre otros para comprobar la factibilidad de un sistema como este y el apoyo que estos brindan al acompañamiento en los emprendimientos.

\section{Limitaciones:}

Esta propuesta por ser relativamente innovadora en el mercado de Costa Rica a nivel privado prevén un arranque lento en su primer año de implementación.

La existencia de muy pocos estudios en relación a la viabilidad de esta línea de negocios a nivel nacional y el desconocimiento del acogimiento que esta propuesta pueda tener a nivel internacional.

\section{Conclusiones}

Después de realizar los estudios de mercado, técnico, organizacional, legal, económico y financiero para conocer la viabilidad de crear una empresa de incubación y aceleración empresarial de carácter bimodal, se concluye que el proyecto es viable, pues la inversión inicial para el proyecto es relativamente baja y se puede optar por varios servicios externos, además se logra un flujo de ingresos aceptables y los indicadores como el VAN y el TIR reflejan resultados positivos.

De acuerdo con autoridades del gobierno, la cultura de emprendimiento es fuerte en el país, pero todavía falta mucho por hacer para mejorar las condiciones a los emprendedores quienes a menudo se ven limitados por obstáculos de financiamiento, legales o falta de apoyo y poder convertir esa energía creativa en empresas exitosas.

A nivel de mercado se determina que es viable, ya que existen proyectos emprendedores que se gestan todos los días.

Por medio del panel experto se pudo determinar la competencia directa, las necesidades y preferencias de las empresas que requieran el servicio. 


\section{Bibliografía}

Cruz Gardner, C. A. \& Matiz Bulla, F. J. (2004) Sistema Nacional de Creación e Incubación de Empresas Servicio Nacional de Aprendizaje Nacional - SENA. Bogotá d.c - Colombia web:, mayo de 2004.

García-Guzmán, M. (2006). tesis "Análisis sobre las potencialidades del uso de la metodología bimodal en la enseñanza de las ciencias sociales, de la Universidad UNIR" (Universidad Internacional de la Rioja) Universidad América, 2006.

González, A. (2015), Emprender con éxito, México Alfa Omega Grupo Editor SA.

Marqués Pere, revista Educación 3.0, la revista para el aula del siglo XXI

Ministerio de Economía, Industria y Comercio (MEIC) (2017). Política Nacional de emprendimiento.

Silva, Jorge, (2013) Emprendedor, hacia un Emprendimiento sostenible Alfa Omega 